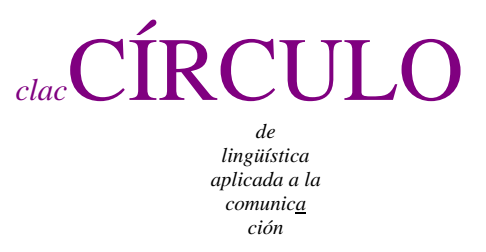

$63 / 2015$

\title{
LA ADQUISICIÓN DEL TIEMPO-ASPECTO EN ESPAÑOL EN L3 PARA LOS APRENDICES TAIWANESES
}

\author{
Hui-Chuan Lu, An Chung Cheng y Sheng-Yun Hung \\ 盧慧娟、鄭安中、洪聖允
}

National Cheng Kung University 國立成功大學

huichuanlu1 en gmail com

\section{Resumen}

Este trabajo tiene como fin investigar el desarrollo de los tiempos pasados en la interlengua de los aprendices taiwaneses que aprenden español como segunda lengua extranjera. El análisis se basa en la Hipótesis de Aspecto Léxico que se centra en la importancia del aspecto léxico de los verbos. Llegamos a las siguientes conclusiones: (1) los aprendices taiwaneses de español cuya lengua materna es chino-mandarín manejan el pretérito mejor que el imperfecto en la producción escrita de los niveles

Hui-Chuan Lu, An Chung Cheng y Sheng-Yun Hung

La adquisición del tiempo-aspecto en español en L3 para los aprendices taiwaneses http://www.ucm.es/info/circulo/no63/lu.pdf

Círculo de Lingüística Aplicada a la Comunicación 63, 200-217.

http://www.ucm.es/info/circulo/no63/barcena.pdf

http://revistas.ucm.es/index.php/CLAC

http://dx.doi.org/10.5209/rev_CLAC.2015.v63.50175

(C) 2015 Elena Bárcena y María Sanfilippo

Círculo de Lingüística Aplicada a la Comunicación (clac)

Universidad Complutense de Madrid. ISSN 1576-4737. http://www.ucm.es/info/circulo 
iniciales de aprendizaje, el desarrollo de adquisición del pretérito indefinido es de acuerdo al siguiente orden: estado, actividad y realización+logro, mientras que para la adquisición del imperfecto los estudiantes siguen un orden contrario. (2) En la mayoría de los casos los aprendices taiwaneses de español siguen el mismo patrón de aprendizaje que los aprendices cuya lengua materna es el inglés en la adquisición del aspecto temporal. Por último, hacemos sugerencias pedagógicas correspondientes a las conclusiones deducidas para mejorar el resultado de aprendizaje. Con esto, esperamos que esta investigación represente una contribución al estudio relacionado con la adquisición del tiempo-aspecto en español.

Palabras clave: tiempo pasado, aspecto léxico, aprendizaje de español, L3

\section{Abstract}

The acquisition of tense-aspect in L3 for Taiwanese speakers of Spanish

The purpose of this study was to investigate the inter-language development of the Spanish past tense by Taiwanese learners who learned Spanish as a second foreign language. The analysis of this study was based on the Lexical Aspect Hypothesis, which focuses on the inherent lexical aspect of verbs. We came to the following conclusions. (1) Taiwanese learners, whose first language was Mandarin Chinese, demonstrated more accurate uses of the Spanish preterite than the imperfect in written texts at their early stages of development. The acquisition of the lexical aspects of verbs in the preterite in terms of lexical aspect demonstrated the following order: achievement + accomplishment, activity and finally stative, whereas the acquisition of the imperfect showed the opposite sequence. (2) In the majority of the cases, the Taiwanese learners of Spanish demonstrated a similar pattern of acquisition of the Spanish past tense in terms of lexical aspect as that of English-speaking learner of Spanish. This paper will end with discussions on pedagogical implications of the findings and we hope this study will shed light on the current research on the second language acquisition of the Spanish tense/aspect.

Key words: past tense, lexical aspect, learning of Spanish, L3 
Índice

1. Introducción 202

2. Antecedentes del estudio 203

2.1. Aspecto gramatical 204

2.2. Aspecto léxico 204

2.3. Adquisición de L2 205

3. Propósito y preguntas del estudio 206

4. Metodología 207

4.1. Participantes y datos 207

4.2. Anotaciones 209

4.3. Cálculo de usos correctos e incorrectos según diferentes variables examinadas 210

5.1. Usos y correcciones de los tiempos pasados 211

5.2. Similitudes y diferencias entre nuestros resultados y los antecedentes 213

6. Implicación pedagógica 214

7. Conclusiones 215

Agradecimientos 215

Referencias bibliográficas 215

Corpus 217

1. Introducción

Este trabajo, tiene como fin investigar el desarrollo del tiempo pasado de la interlengua de los aprendices taiwaneses que aprenden inglés como segunda lengua. El análisis 
realizado, se basa en la Hipótesis de Aspecto, que se centra en la importancia del aspecto léxico, para la adquisición de la morfología temporal. El chino-mandarín, es una lengua que no marca el tiempo mediante la inflexión morfológica, tal como es el rasgo [+/-pasado]. Dicho rasgo, está ausente en la lengua materna (el chino-mandarín) de los aprendices taiwaneses. Por tal razón, esta investigación representa una contribución al estudio de la adquisición del tiempo-aspecto en español. Nuestro estudio se organiza de la siguiente forma : empezaremos con una breve introducción sobre el trabajo; en la primera sección, repasaremos los antecedentes de otros estudios relacionados; presentaremos el propósito y las preguntas de estudio, la metodología y los resultados en la segunda, tercera y cuarta seccion respectivamente. Luego, la quinta sección se dedicará a la implicación pedagógica. Por último, concluiremos la investigación en la sección siete.

\section{Antecedentes del estudio}

La mayor diferencia entre las lenguas europeas y el idioma chino se deriva de la expresión verbal. En las lenguas indoeuropeas tales como el inglés o el español, se expresan los tiempos presente, pasado y futuro así como los aspectos progresivos y perfectivos mediante las inflexiones verbales. El tiempo indica en la línea temporal la posición del presente, pasado o futuro, con respecto a la relación relativa entre un evento y un tiempo. Sin embargo, el aspecto define la característica interna temporal de un evento expresando el punto de vista del hablante sin asociarse con la línea temporal (Comrie, 1976). El inglés se parece al español en cómo se expresa el orden temporal y el aspecto, a través de la inflexión verbal. No obstante, en chino no existen las conjugaciones morfológicas para distinguir los diferentes tiempos y los aspectos. Los tiempos y aspectos se expresan por los adverbios temporales, léxicos, discurso, semántica y pragmática contextual, y los marcadores aspectuales. Por lo tanto, los aprendices taiwaneses, cuya lengua materna es chino-mandarín, deben cambiar su costumbre para dominar los tiempos y aspectos (qué aspectos), aprendiendo las maneras de expresarlos en español aplicando la forma morfológica verbal apropiada.

En esta sección incluiremos antecedentes de estudios provenientes de tres direcciones, el aspecto gramatical, el léxico y la adquisición de L2. 


\subsection{Aspecto gramatical}

El aspecto gramatical en inglés y en español se expresa mediante el verbo auxiliar y la inflexión verbal para distinguir las diferencias entre lo perfectivo y lo imperfectivo. En los tiempos pasados, los marcadores temporales y aspectuales, pueden señalar la distinción entre ellos. No obstante, los aspectos gramaticales no se determinan por el sentido léxico del predicado verbal, porque un verbo se puede conjugar en más de un tipo de desinencia flexional. Comrie (1976) indica que para un mismo hablante, se puede usar el imperfecto o el pretérito para un mismo contexto sin generar ningún conflicto entre ellos. En contraste con el español, el chino-mandarín no marca el tiempo para la morfología verbal, sino utiliza cuatro marcadores aspectuales, GUO, ZAI, ZHE y $L E$. En general, el chino-mandarín distingue los aspectos gramaticales por adverbios, perífrasis adverbiales, argumentos y los marcadores contextuales.

\subsection{Aspecto léxico}

Con respecto a las teorías de aspectos léxicos, Vendler (1967) indica que el estado, la actividad, la realización y el logro son cuatro tipos básicos y se distinguen por el sentido léxico interno de los verbos o los predicados verbales. Los verbos que expresan los estados son estáticos, permanecen sin sufrir cambio alguno, por ejemplo, los verbos “amar, odiar, esperar”. Los verbos de “actividad” indican las acciones durativas, tales como “comer, cantar y bailar”, que pueden discontinuar arbitrariamente y los diferentes segmentos de una acción referirse a un mismo evento. Los verbos de realización son los que identifican las acciones durativas que poseen un final claro de acción terminada, como “construir una casa, correr un kilómetro” etc. Los verbos de logro son los que identifican las acciones instantáneas, no-durativa y tiene un final, por ejemplo, “morir, encontrar, perder, ganar”. Además, Comrie (1976) y Andersen (1991) usan tres rasgos semánticos (dinámico, télico y puntual) para distinguir los aspectos verbales. Los verbos de realización son los que identifican acciones télicas y los verbos de logro son los que identifican acciones instantáneas, mientras que los verbos de estado difieren de los de actividad, realización y logro. Cualquier verbo no se limita a un solo aspecto léxico específico, porque los valores de las clasificaciones de los predicados verbales siempre varían según el sentido interno y los argumentos externos. 


\subsection{Adquisición de L2}

En el marco de la adquisición de L2, nos basamos en la Hipótesis de Aspecto Léxico (HAL) de Andersen (1991) para explicar las relaciones entre los morfemas verbales y los tipos de verbos léxicos. Para HAL, en la etapa inicial de la adquisición de lengua, la forma en que los aprendices hacen uso de los aspectos temporales se fundamenta en los aspectos léxicos inherentes. En otras palabras, los principiantes no codifican los aspectos gramaticales ni los temporales. Andersen (1991) propone que existe un proceso gradual en el desarrollo de la adquisición de L2: verbo puntual $\rightarrow$ télico $\rightarrow$ dinámico $\rightarrow$ estático. Él apunta el proceso de desarrollo para los aprendices de los tiempos españoles en base de las interacciones entre las derivaciones verbales de 2 aspectos, 4 clases léxicas y 8 etapas de desarrollo, como se señala en el Cuadro 1.

Cuadro 1. Orden de adquisición

\begin{tabular}{|l|l|l|l|l|}
\hline Nivel & Estado & Actividad & Realización & Logro \\
\hline 1 & Presente & Presente & Presente & Presente \\
\hline 2 & Presente & Presente & Presente & Pretérito \\
\hline 3 & Imperfecto & Presente & Presente & Pretérito \\
\hline 4 & Imperfecto & Imperfecto & Pretérito & Pretérito \\
\hline 5 & Imperfecto & Imperfecto & $\begin{array}{l}\text { Pretérito/ } \\
\text { Imperfecto }\end{array}$ & Pretérito \\
\hline 6 & Imperfecto & $\begin{array}{l}\text { Pretérito/ } \\
\text { Imperfecto }\end{array}$ & $\begin{array}{l}\text { Pretérito/ } \\
\text { Imperfecto }\end{array}$ & Pretérito \\
\hline 7 & Imperfecto & $\begin{array}{l}\text { Pretérito/ } \\
\text { Imperfecto }\end{array}$ & $\begin{array}{l}\text { Pretérito/ } \\
\text { Imperfecto }\end{array}$ & $\begin{array}{l}\text { Pretérito/ } \\
\text { Imperfecto }\end{array}$ \\
\hline 8 & $\begin{array}{l}\text { Pretérito/ } \\
\text { Imperfecto }\end{array}$ & $\begin{array}{l}\text { Pretérito/ } \\
\text { Imperfecto }\end{array}$ & $\begin{array}{l}\text { Pretérito/ } \\
\text { Imperfecto }\end{array}$ & $\begin{array}{l}\text { Pretérito/ } \\
\text { Imperfecto }\end{array}$ \\
\hline
\end{tabular}

En general, para los aprendices que aprenden español como L2, en el proceso de desarrollo, el pretérito se adquiere más temprano que el imperfecto. Para los aprendices de español cuya lengua materna es inglés, el uso del pretérito indefinido se considera como el uso predeterminado (Lafford, 1996; Liskin-Gasparro, 2000; Salaberry, 1999). Además, los resultados de Salaberry (2003) indican que la HAL se aplica mejor para los estudiantes de niveles más avanzados: el 63\% de los usos del imperfecto se asocian con los verbos de estado, 
y el 82\% de los usos del pretérito se relacionan con los verbos télicos. Los pretéritos se usan con más frecuencia junto con las acciones télicas y raras veces se asocian con los verbos estáticos. Además, el imperfecto se usa con mayor frecuencia con los verbos estáticos, y menos con las acciones télicas. Por lo tanto, los aprendices del nivel intermedio se tienden a utilizar el pretérito en todos los tipos de verbos.

Salaberry (2003) concluye que en la etapa inicial de los aprendices cuya lengua materna es inglés, parece que el tiempo español (o sea, la influencia del aspecto gramatical de la desinencia verbal) sobrepasa la del aspecto léxico. Sin embargo, la influencia léxica crece a lo largo de la adquisición de lengua. El estudio de Ramsay (1990) apoya la HAL, el desarrollo continuo mencionado anteriormente sólo se encuentra en los casos que distinguen los verbos estáticos y los télicos, sin poder diferenciar los aspectos verbales de realización y logro. Liskin-Gasparro (2000) propone que las variables que afectan las selecciones de usos en los dos tiempos pasados se asocian con la léxica, el discurso, la enseñanza, el trabajo etc. De todos modos, lo más importante es que el pretérito sigue siendo el uso preestablecido.

Para ampliar la aplicación del marco teórico con respecto a la adquisición de la lengua romance como la segunda lengua, en la adquisición de tiempos y aspectos, Salaberry (2003) y Dalila and Salaberry (2005) sugieren que los futuros estudios deben tomar en cuenta a los aprendices asiáticos. Como el presente estudio se basa en un sub-corpus que recopila los datos de los participantes taiwaneses, esperamos que los resultados puedan enriquecer los conocimientos actuales sobre aspectos temporales en la adquisición de L2.

\section{Propósito y preguntas del estudio}

En el área semántica, a fin de verificar la aplicación de la Hipótesis del Aspecto Léxico para los aprendices taiwaneses y enriquecer los resultados de las teorías relacionadas, investigaremos el desarrollo de la adquisición de dos aspectos en el idioma español que son el pretérito indefinido y el imperfecto, estudiando así la distribución en la que los estudiante usan dichos verbos, y a su vez analizando su tendencia de uso, en contraste con la de los hablantes nativos.

Según nuestro propósito de investigación, planteamos las siguientes preguntas de estudio:

(1) ¿Cuál es el modelo de desarrollo para la adquisición de los tiempos pasados para los aprendices taiwaneses quienes aprenden español como segunda lengua extranjera? 
a. A lo largo del desarrollo, ¿̇mejoran los estudiantes su uso del pretérito indefinido, en un orden jerárquico, en los verbos télicos, dinámicos y estáticos?

b. A lo largo del desarrollo, ¿mejoran los estudiantes su uso del imperfecto, en un orden jerárquico, en los verbos estáticos, dinámicos y télicos?

(2) ¿Son iguales los modelos para los aprendices taiwaneses cuya lengua materna es chino-mandarín y los aprendices cuya lengua materna es inglés?

\section{Metodología}

\subsection{Participantes y datos}

En el presente estudio transversal, analizaremos los datos recogidos en el año 2010 y compilados en el CEATE (Corpus Escrito de Aprendices Taiwaneses de Español), anotando las clasificaciones de verbos léxicos a fin de examinar el modelo de adquisición de los tiempos verbales para los aprendices taiwaneses de diferentes niveles, seguidamente hacemos una comparación de los resultados concluidos con los resultados obtenidos en estudios anteriores. La creación de CATE (Corpus de Aprendices Taiwaneses de Español) ha durado seis años (2005-2010). Se han recogido 2.443 textos escritos, aproximadamente 442.000 .000 palabras, anotadas con errores-correcciones, partes de la oración y lema. Ya se puede consultar públicamente los datos de los primeros dos años (2005-2006) a través del sitio web http://corpora.flld.ncku.edu.tw. Además, se puede consultar una palabra específica, su POS, la siguiente POS y un lema verbal. Los datos que pueden consultarse públicamente se limitan a los recopilados entre 2005-2007.

Hemos compilado 49.606 palabras de 222 textos procedentes de seis universidades (TKU, PU, WZUC, NCKU, NCU y HGTU). Los participantes tardaron 45 minutos para realizar la evaluación de español, Wisconsin Placement Test of Spanish. Según los resultados de dicha evaluación, la capacidad de dominio del idioma español se clasifica en 5 niveles, en base al estándar establecido por varias universidades estadounidenses: nivel I (0-27 respuestas correctas), nivel II (28-48 correctas), nivel III (49-58 correctas), nivel IV (59-67correctas) y nivel V (68-76 correctas). (Los niveles IV y V no se incluyen en el posterior análisis porque contienen menos de 10 personas.) 
Durante la realización, los participantes deben narrar el cuento de Caperucita Roja utilizando los dos tiempos pasados según las ilustraciones ofrecidas sin hacer uso de diccionario ni cualquier otro instrumento auxiliar (véanse las Figuras 1 y 2).

Figuras 1 y 2
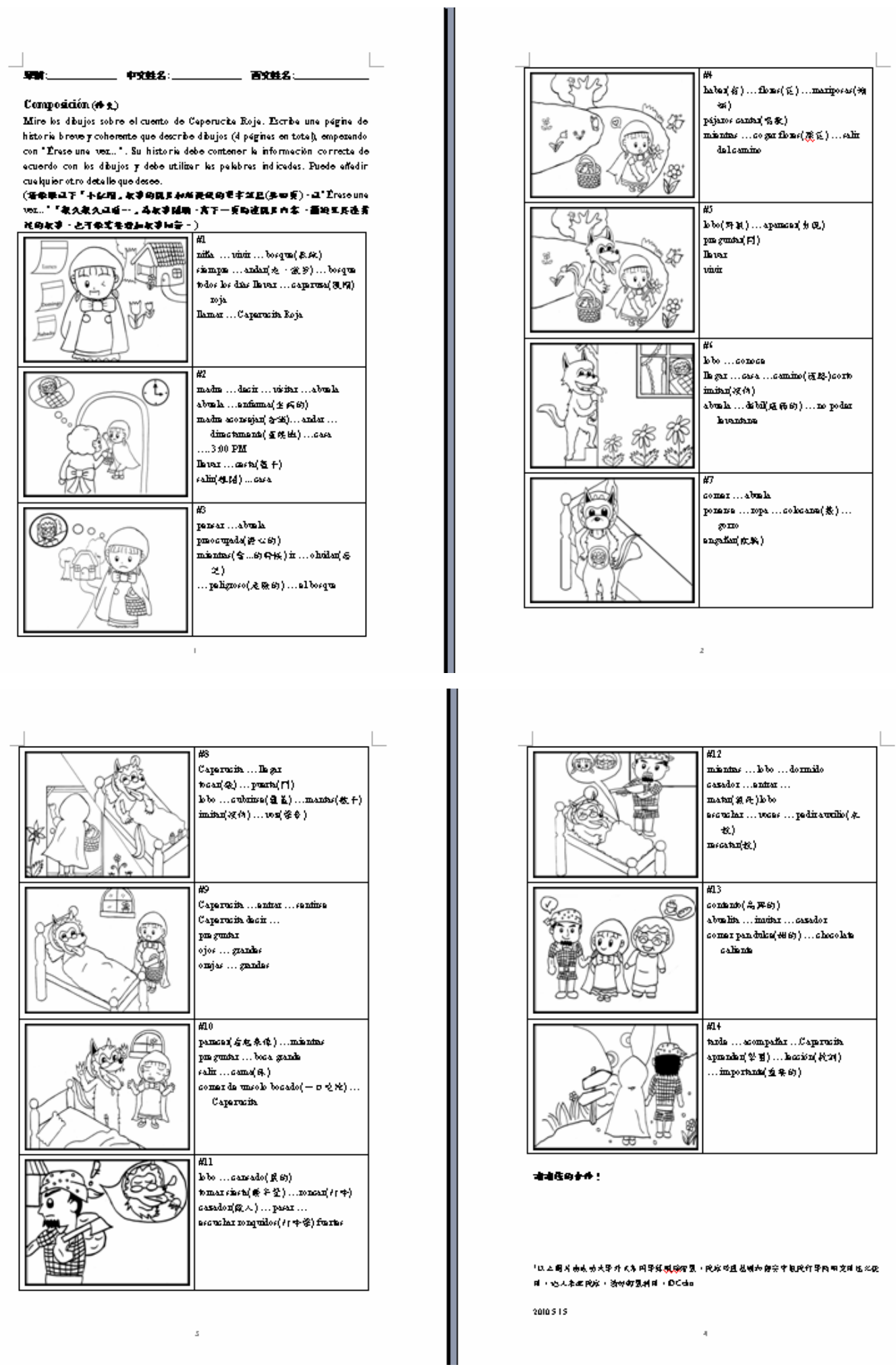
Por la razón de representatividad, los participantes que se evalúan en los niveles IV y V y los textos escritos con menos de 100 palabras no se incluyen en el análisis posterior. Por fin, anotamos 143 textos, 33.655 palabras. (11 del nivel I, 103 del nivel II y 29 del nivel III), como se observa en el Cuadro 2.

Cuadro 2. Textos y palabras de diferentes niveles

\begin{tabular}{|l|l|l|l|l|}
\hline Nivel & I & II & III & Total \\
\hline Respuestas cor. & $0 \sim 27$ & $28 \sim 48$ & $49 \sim 58$ & \\
\hline Textos & 11 & 103 & 29 & 143 \\
\hline Palabras & 2.156 & 23.054 & 8.445 & 33.655 \\
\hline
\end{tabular}

\subsection{Anotaciones}

Anotamos los usos de los tiempos pasados aparecidos en los textos escritos por nuestros participantes, en contraste con los textos revisados por los hablantes nativos del idioma español, utilizando el Corpus Tool de UAM como la herramienta auxiliar para facilitar el trabajo de anotaciones con respecto a las variables examinadas, en total realizamos 7.481 anotaciones incluyendo las siguientes categorías. (a) Las inflexiones verbales de los usos tanto de los aprendices como de los hablantes nativos: se clasifican en los usos correctos e incorrectos contrastando los usos nativos y los usos de los aprendices. Además, se agrupan en: el presente, el pretérito indefinido, el pretérito imperfecto, el infinitivo, el participio pasado etc. (b) Los aspectos léxicos de los verbos usados: según el sentido interno del léxico, se clasifican en (i) estado (acción estática), (ii) actividad (acción durativa e atélica) y (iii) realización (acción durativa y télica) y logro (acción no-durativa y télica). Los verbos ofrecidos, acompañados con las ilustraciones se presentan en el Cuadro 3 y son distribuidos según sus categorías léxicas. Por ejemplo, “vivir, andar y pedir auxilio” pertenecen a la categoría de estado, actividad y logro respectivamente. 
Cuadro 3. Clasificaciones léxicas de verbos

\begin{tabular}{|l|l|l|l|l|}
\hline Tipos & Estado & Actividad & Realización & Logro \\
\hline Pretérito & sentirse & $\begin{array}{l}\text { llevar una } \\
\text { cesta, salir, } \\
\text { imitar, roncar, } \\
\text { invitar, comer } \\
\text { pan dulce... }\end{array}$ & $\begin{array}{l}\text { decir, aconsejar, } \\
\text { preguntar, } \\
\text { ponerse ropa, } \\
\text { colocarse gorro, } \\
\text { tomar siesta, } \\
\text { engañar, pasar, } \\
\text { aprender }\end{array}$ & $\begin{array}{l}\text { olvidar, salir } \\
\text { de, aparecer, } \\
\text { llegar, } \\
\text { comer...abuela, } \\
\text { tocar, cubrirse, } \\
\text { entrar, comer } \\
\text { de un solo } \\
\text { bocado, } \\
\text { escuchar, } \\
\text { rescatar }\end{array}$ \\
\hline Imperfecto & $\begin{array}{l}\text { vivir, estar, ser, } \\
\text { haber, conocer, } \\
\text { poder, parecer }\end{array}$ & $\begin{array}{l}\text { andar, llamar, } \\
\text { ir, cantar, coger } \\
\text { flores, pensar }\end{array}$ & & pedir auxilio \\
\hline
\end{tabular}

4.3. Cálculo de usos correctos e incorrectos según diferentes variables examinadas

Para contestar las dos preguntas de estudio, nos centramos en el análisis de los dos tiempos pasados, el pretérito indefinido y el imperfecto, considerando tanto los usos y las correcciones de las formas objetivas seleccionadas por los aprendices. En el cálculo de corrección, aprovechando la función contrastiva que poseen el corpus CEATE, nos es fácil calcular los usos correctos e incorrectos mediante las comparaciones y los contrastes entre los usos de los aprendices y los hablantes nativos que se encargan de revisar los textos recopilados consultándolos con los resultados anotados. Tomando como ejemplo los usos del pretérito indefinido, la corrección se calcula como: los usos correctos del pretérito indefinido utilizados por los aprendices se dividen entre los usos del pretérito indefinido utilizados por los hablantes nativos del idioma español:

los usos correctos del pret. utilizados por los aprendices

los usos del pret. utilizados por los nativos del esp.

Con el fin de observar la tendencia de usos en diferentes etapas de adquisición, calculamos las correcciones según diferentes variables mencionadas anteriormente.

\section{Resultados y debate}

A continuación se señalan los resultados con respecto a las correcciones por un lado y las similitudes y diferencias entre nuestros resultados y los antecedentes por otro. 
5.1. Usos y correcciones de los tiempos pasados

Los resultados de corrección según diferentes niveles se señalan en los Diagramas 1 y 2.

Diagrama 1. Tendencia de adquisición en dos aspectos

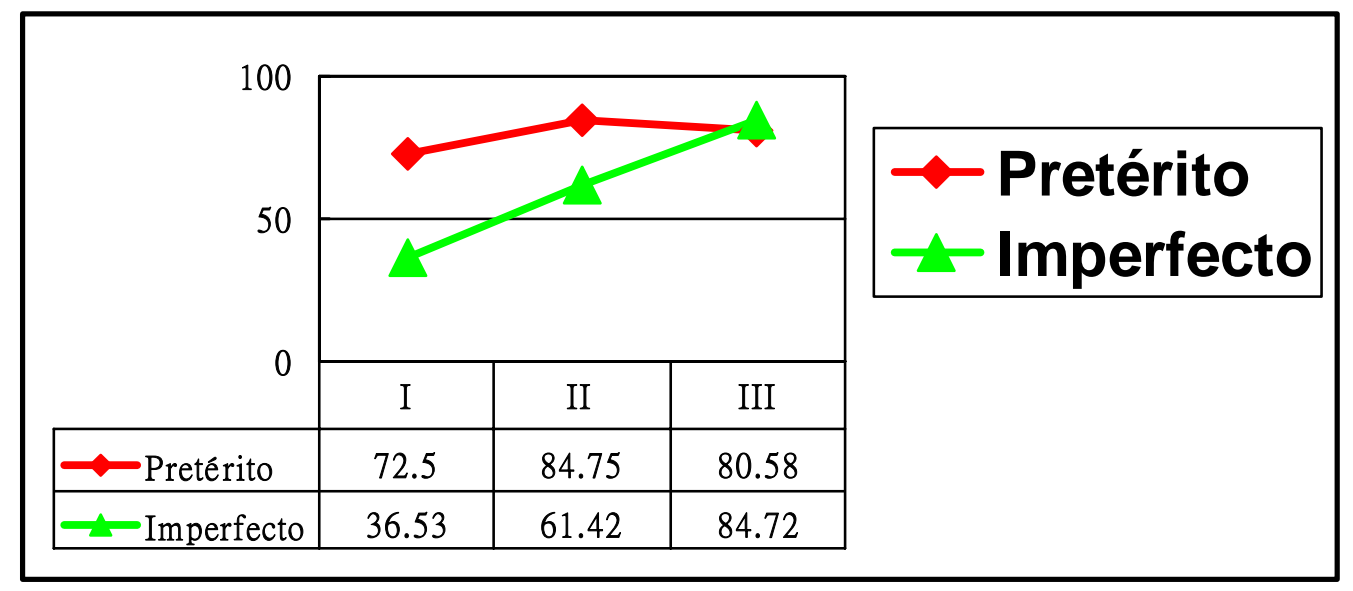

Según el Diagrama 1, podemos observar que las correcciones de los usos en el pretérito superan a las correcciones de los usos en el imperfecto para los primeros dos niveles (I y II), mientras que el número de correcciones de los usos del imperfecto en el nivel III excede el de los usos del pretérito. Esto indica que los principiantes taiwaneses cuya lengua materna es chino manejan mejor los usos del pretérito que los del imperfecto en el desarrollo de la adquisición.

Avanzando un paso más, los resultados entre diferentes niveles, aspectos gramaticales y categorías léxicas se muestran en el Diagrama 2.
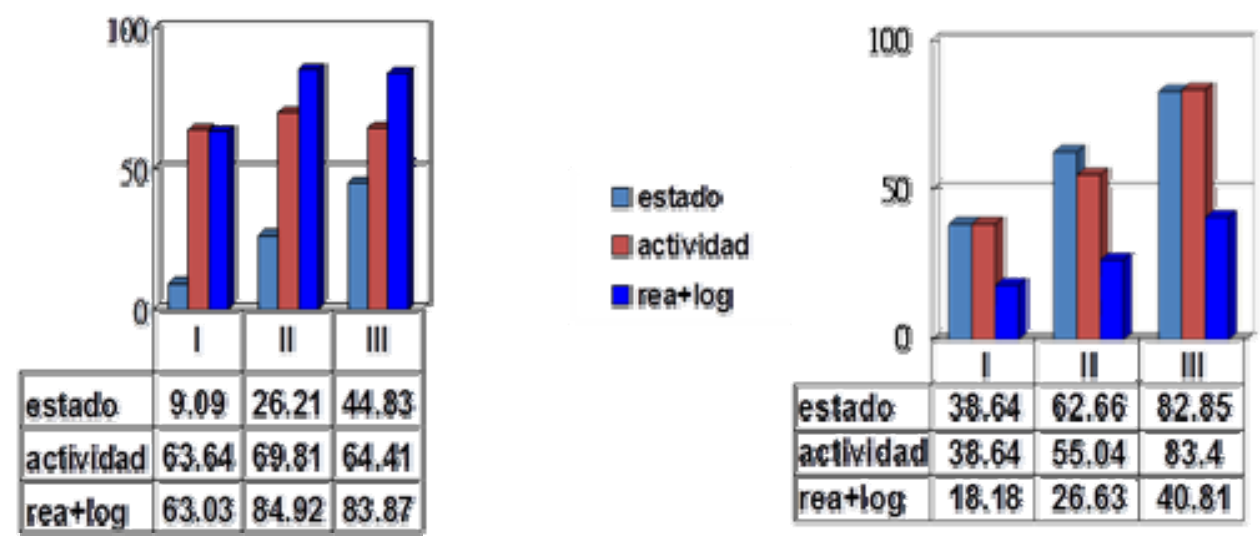

Diagrama 2. Tendencia de desarrollo según diferentes categorías léxicas 
En el Diagrama 2, observamos los siguientes resultados. En primer lugar, en el uso del pretérito, los aprendices del primer nivel usan las clases de verbos de acuerdo a la siguiente jerarquía de corrección (estado, 9,09\%<logro+realización, 63,03\%<actividad, 63,64\%) para el aspecto del pretérito, mientras que los verbos de estado (38,64\%) y los de actividad (38,64\%) igualmente se usan más correctamente que los télicos (realización+logro, 18,18\%) en el caso del imperfecto. A pesar de que las correcciones de los verbos de actividad (63,64\%) en los usos del pretérito superan a las de los télicos (63,03\%), dicha diferencia es muy poca. En segundo lugar, los aprendices del nivel II, se comportan diferentemente en los dos aspectos. Por un lado, en los usos del pretérito, ellos controlan los verbos de actividad (69,81\%) mejor que los de estado $(26,21 \%)$ y seleccionan más correctamente los verbos télicos (84,92\%) que los de actividad (69,81\%). Por otro lado, en el uso del imperfecto, las correcciones siguen un tendencia contraria: estado (62,66\%) >actividad (55,04\%) >logro+realización (26,63\%). Por último, se observa que los aprendices del nivel III se parecen a los del nivel II en los usos del pretérito (logro+realización, 83,87\%> actividad, 64,41\%>estado, 44,83\%). Aunque se distinguen en que los aprendices del nivel III utilizan los verbos siguiendo el orden de corrección de actividad $(83,4 \%)$ > estado (82,85\%) >realización+logro (40,81\%), se observan que las diferencias entre actividad y estado son pequeñas (0,55\%). Los correspondientes ejemplos de los aprendices son mostrados en las oraciones (1)-(10).

Usos incorrectos

Nivel I:

(1) Actividad (Pretérito): ...siempre andé con bosque todos los días...( El uso correcto debe ser “Siempre andaba por el bosque todos los días...”.) (\#10013)

(2) Actividad (Imperfecto): ...lobo cubrirse la mantas y imitiá voz de abuela. (El uso correcto debe ser “...lobo estaba cubierto con la mantas e imitó la voz de la abuela.”) (\#10023)

Nivel II:

(3) Estado (Pretérito): El lobo conoció a la casa de abuela...( El uso correcto debe ser “El lobo conocía la casa de la abuela...”.) ( \#10038)

(4) Realización+Logro (Imperfecto): El lobo aparecía y preguntó...( El uso correcto debe ser “Un lobo apareció y preguntó...”. ( \#10097) 


\section{Usos correctos}

Nivel I:

(5) Realización+Logro (Pretérito): Caperucita aprendió un lección muy importante. (\#10126)

(6) Estado (Imperfecto): Érase una vez, una niña vivía en el bosque. (\#10203)

Nivel II:

(7) Actividad (Pretérito): ...el cazador acompañó a Caperucita...( \#10062)

(8) Actividad (Imperfecto): Siempre andaba en el bosque. (\#10097)

Nivel III:

(9) Estado (Pretérito): abuela estaba muy débil, no pudo levantarse y... (\#10054)

(10) Realización+Logro (Imperfecto): hay un cazador escuchó los voces pedían auxilios,...( \#10114)

Los resultados concluidos dan repuesta a la primera pregunta de estudio: los aprendices taiwaneses de español cuya lengua materna es chino manejan el pretérito mejor que el imperfecto en los niveles iniciales, y siguen un quasi-orden de estado-actividadrealización+logro, en el desarrollo de adquisición, realización+logro>actividad>estado en los usos del pretérito y el orden contrario en los usos del imperfecto. O sea, a lo largo del desarrollo, se aumentan los correctos usos del pretérito indefinido de un orden jerárquico en los verbos télicos, dinámicos y estáticos mientras que se aumentan los correctos usos del imperfecto de un orden jerárquico en los verbos estáticos, dinámicos y télicos en la mayoría de casos estudiados.

\subsection{Similitudes y diferencias entre nuestros resultados y los antecedentes}

Un paso más adelante, hacemos comparaciones y contrastes con los resultados de los estudios anteriores relacionados con los aspectos léxicos. Según los estudios anteriores (Lafford, 1996; Liskin-Gasparro, 2000; Salaberry, 1999), los aprendices de español cuya lengua materna es el inglés establecen el pretérito indefinido con el valor predeterminado en la selección de los tiempos pasados, mientras que los aprendices del nivel intermedio tienden a utilizar el pretérito para todos los tipos de aspecto léxico. Esto concuerda con nuestro resultado, a pesar de las diferentes metodologías adoptadas, los aprendices de niveles iniciales presentan el mismo comportamiento. Con esta información, podemos responder la segunda pregunta del estudio 2: ¿Son iguales los 
modelos para los aprendices taiwaneses cuya lengua materna es chino-mandarín y los aprendices cuya lengua materna es inglés? Este estudio, indica que, para los aprendices taiwaneses, la adquisición del aspecto temporal, según las categorías léxicas es bastante parecida a los resultados de los aprendices cuya lengua materna es inglés.

\section{Implicación pedagógica}

En la aplicación de enseñanza, sugerimos que en la etapa inicial del aprendizaje, después de que se hayan familiarizado con las desinencias inflexionales, los profesores pueden empezar la enseñanza de los tiempos pasados con las actividades de los verbos télicos para mejorar los resultados, porque el uso del pretérito en las actividades generalmente se considera como la forma para narrar todos los eventos o experiencias. En el siguiente paso, los aprendices pueden practicar el imperfecto con los verbos de estado o actividad.

Para integrar los resultados estudiados a la enseñanza, examinamos los textos usados en Taiwán concentrándonos en los dos aspectos diferentes. Nos damos cuenta de que todos los textos empiezan con el pretérito y después, el imperfecto, como se han indicado en los estudios anteriores. Sin embargo, la única categoría léxica que se menciona es el estado y las demás categorías tales como la actividad, la realización o el logro no se distinguen claramente. Es de gran importancia el clarificar las categorías léxicas de los verbos y enfatizar su asociación con los aspectos seleccionados para mejorar el resultado de adquisición eficientemente.

Por último, sugerimos que los enseñantes aprovechen los recursos de córpora existentes tales como el Corpus del Español (CdE) y el Corpus Escrito de Aprendices Taiwaneses de Español (CEATE) para diseñar los materiales didácticos adecuados. El primer corpus, CdE (http://www.corpusdelespanol.org/) ofrece auténticos y abundantes ejemplos de contexto, de los nativos hispánicos. Con el segundo corpus, CEATE (http://corpora.flld.ncku.edu.tw/), se puede consultar los datos para hacer los ejercicios de análisis contrastivo entre los usos incorrectos y correctos, los errores escritos por los aprendices taiwaneses y corregidos por los nativos hispánicos, respectivamente. Así que los aprendices no practican mecánicamente las conjugaciones verbales, sino que los profesores pueden diseñar actividades pedagógicas considerando las características léxicas de aspectos verbales para adecuarlos a los niveles de los aprendices. 


\section{Conclusiones}

Este trabajo, tiene como fin el investigar el desarrollo de los tiempos pasados en la interlengua de los aprendices taiwaneses que aprenden español como segunda lengua. El análisis realizado, se basa en la Hipótesis de Aspecto, y llegamos a las siguientes conclusiones: (1) los aprendices taiwaneses de español cuya lengua materna es chino manejan el pretérito mejor que el imperfecto en los niveles iniciales de aprendizaje, el desarrollo de adquisición del pretérito indefinido se hace de acuerdo al siguiente orden: estado<actividad<realización+logro, Mientras que para la adquisición del imperfecto los estudiantes siguen un orden contrario. (2) En la mayoría de los casos, en la adquisición del aspecto temporal, los aprendices taiwaneses de español siguen el mismo patrón de aprendizaje que los aprendices cuya lengua materna es el inglés.

Por último, hicimos sugerencias pedagógicas correspondientes a las conclusiones deducidas para mejorar el resultado de aprendizaje. Con esto, esperamos que esta investigación pueda arrojar luz en el estudio relacionado con la adquisición del tiempoaspecto en español.

Agradecimientos

Agradecemos por el apoyo financiero del siguiente proyecto de investigación: NSC992410-H-006-092-MY2. Además, damos gracias por la ayuda de los siguientes profesores: Wen-Hsian Lu, Lucía Lu, Paloma, Pai, Delia Lin, Teresa Cheng, Emilia Cheng, Edelmira Mao, Azucena Lin. Por último, nos gustaría expresar nuestro sincero agradecimiento a todos los asistentes y participantes que han colaborado en el presente estudio.

Referencias bibliográficas

Andersen, R. (1991). Developmental sequences: The emergence of aspect marking in second language acquisition. In T. Huebner \& C.A. Ferguson (Eds.), Crosscurrents in second language acquisition and linguistic theories (pp. 30524). Amsterdam: John Benjamins. 
Comrie, B. (1976). Aspect. Cambridge: Cambridge University Press.

Dalila, A. \& Salaberry, M. R. (2005). Tense and aspect in romance languages. Amsterdam: John Benjamins.

Lafford, B. (1996). The development of tense/aspect relations in L2 Spanish narratives: Evidence to test competing theories. Paper presented at the Second language Research Forum. Tucson.

Liskin-Gasparro, J. (2000). The acquisition of temporality in Spanish oral narratives: Exploring learners’ perceptions. Hispania, 830-44.

Lu, Hui-Chuan \& Cheng, An-Chung. (2010, November). Corpus-based study on third language acquisition of Spanish past tense and aspect. Paper presented at the International Conference on Applied Linguistics, Chiayi, Taiwan.

Lu, Hui-Chuan \& Cheng, An Chung. (2011, July). La adquisición del tiempo-aspecto en L3 para los aprendices taiwaneses, comunicación presentada en el XLVI Congreso Internacional de la AEPE, Cuenca.

Lu, Hui-Chuan \& Cheng, An Chung. (2011). 語料庫㷇本之西語時態動貌習得研究.

[Investigación sobre la adquisición del tiempo-aspecto basada en un corpus de español], comunicación presentada en el XII Congreso Nacional de PU: Lengua, Literatura y Cultura en el Mundo Hispánico, Taichong, Taiwan.

Lu, Hui-Chuan, Cheng, An Chung \& Hung, Shen-Yun. (2014). “Creation of CATE and a Corpus-based Study on L3 Acquisition of the Spanish Past Tense”. $11^{\text {th }}$ Teaching and Language Corpora Conference (TaLC11). Lancaster.

Ramsay, V. (1990). Developmental stages in the acquisition of the perfective and the imperfective aspects by classroom L2 learners of Spanish. Tesis doctoral. University of Oregon.

Salaberry, R. (1999). Procedural vocabulary: Lexical signaling of conceptual relations in the development of past tense verbal morphology in classroom L2 Spanish. Applied Linguistics, 20, 151-178.

Salaberry, R. (2003). Tense aspect in verbal morphology. Hispania, 86, 559-573.

Vendler, A. (1967). Linguistics in philosophy. Ithaca, NY: Cornell University Press. 
lu, cheng y hung: tiempo-aspecto 217

\section{Corpus}

CEATE: Corpus Escrito de Aprendices Taiwaneses de Español http://corpora.flld.ncku.edu.tw

CdE: Corpus del Español http://www.corpusdelespanol.org/

UAM Corpus Too: http://www.wagsoft.com/CorpusTool/

Recibido: 3 de septiembre de 2014

Revisado 9 de noviembre de 2014

Aceptado: 16 de junio de 2015

Publicado: 28 de septiembre de 2015 